\title{
Robust Phase Tracking for High Capacity Wireless Multimedia Data Communication Networks
}

\author{
Taehyun Jeon \\ Seoul National University of Technology, \\ Dept. of Electrical Engineering, \\ 172 Gongneung-2Dong, Nowon-Gu \\ 139-743 Seoul, Korea \\ thjeondsnut.ac.kr
}

\begin{abstract}
This paper proposes an algorithm for tracking of the residual phase errors incurred by carrier and sampling frequency offset in the OFDM transmission systems which is suitable for high speed and high capacity wireless multimedia communication networks. The proposed scheme utilizes the degree of the channel fading in the frequency domain in the offset tracking procedure which improves the error estimation accuracy and the tracking performances. This scheme also contributes to the increase of the throughput and the network capacity by reduction of the packet error rate or equivalently probability of re-transmission events which are critical QoS requirements for multimedia data transmissions.
\end{abstract}

Keywords: ubiquitous network, QoS, carrier frequency offset, OFDM.

\section{Introduction}

The demands for access to the high quality multimedia data any time any where have been increased dramatically with the introduction of the concept of ubiquitous networks. The requirements for such a high capacity data transmission include enhanced data retrieval under various channel and interference environments as well as the data rate increase up to hundreds or even to gigabits per second ranges depending on the application areas. The orthogonal frequency division multiplexing (OFDM) system achieves high data rates by simultaneous transmission of multiple data symbols through subcarriers which are orthogonal to each others [1]. As in the general synchronous digital communication systems, the carrier frequency difference between transmitter and receiver sides in the OFDM plays an important role in the entire system performance. Another important impairment is the sampling frequency offset which is generated in the process of digital-to-analog (D/A) and analog-todigital (A/D) conversion in both sides, respectively [2].

This paper proposes a reliable estimation and tracking method for phase errors based on the channel information in the OFDM systems. In this scheme the received signal processed by the frequency domain equalizer is used for the error estimation and the channel gain for each subcarrier is utilized to provide the improved reliability. 


\section{Phase Error Detectors}

In this section the detection methods for phase errors are described which are caused by the local oscillators' carrier frequency difference and sampling clock frequency difference at transmitter and receiver sides. For better understanding of the proposed algorithm one of the widely used OFDM based local area wireless data transmission systems is assumed [3].

\subsection{Phase Errors by Carrier Frequency Offset}

The phase error caused by carrier frequency offset (CFO) can be approximated as the same for all subcarriers in the frequency domain within one OFDM symbols. Here the CFO phase error can be estimated as the average value over all corresponding subcarriers within one symbol period. In case only the pilot subcarriers are utilized for the error estimation the estimated phase error by CFO is represented as follows:

$$
\hat{\theta}_{i}=\frac{1}{N_{p}} \sum_{j \in\{\text { pilot_index }\}} \phi_{i, j}
$$

where pilot_index represents a set of subcarrier indices where the pilots are located and $N_{p}$ the total number of pilot subcarriers within one OFDM symbol. Also $\phi_{i, j}$ is the estimated phase error values contained in the received signal sample where $i$ and $j$ represent indices for OFDM symbol and subcarrier, respectively.

When payload signals are also used for the phase error estimation the error detector uses slightly modified procedure considering the locations and the involved number of data subcarriers. The combined form of phase error estimation can be represented as follows when both the pilot and the payload data with channel quality information are utilized:

$$
\hat{\theta}_{i}=\frac{\sum_{j \in\{\text { pilot_index }\}}\left|\hat{H}_{j}\right|^{2} \phi_{i, j}+\sum_{j \in\{\text { data_index }\}}\left|\hat{H}_{j}\right|^{2} \phi_{i, j}^{\prime}}{N_{p} \sum_{j \in\{\text { pilot_index }\}}\left|\hat{H}_{j}\right|^{2}+N_{d} \sum_{j \in\{\text { data_index }\}}\left|\hat{H}_{j}\right|^{2}}
$$

where the data_index represents a set of indices where payload data are located. Also $N_{d}$ is the total number of subcarriers associated with payload data and $\phi_{i, j}^{\prime}$ is the estimated phase error based on data subcarriers. The estimated channel gain in $j^{\text {th }}$ subcarrier is represented as $\hat{H}_{j}$ which is obtained using the preamble part appeared at the beginning portion of the packet. The estimation performance is expected to be improved when the channel information for each subcarrier is involved in the phase error estimator. In that case the channel with higher gain contributes more in the 
estimation process while the channel experiencing more fading less contribution which can provide more reliable results.

\subsection{Phase Errors by Sampling Frequency Offset}

The presence of sampling frequency offset in the time domain is reflected into the linearly varying phase error in the frequency domain. Here the slope of the phase error in the frequency domain corresponds to the sampling frequency offset. Detection of the slope based on the pilot subcarriers can be summarized as follows when the well known linear regression method is applied:

$$
\hat{\alpha}_{i}=\frac{\sum_{j \in\{\text { pilot_index }\}} j \cdot \phi_{i, j}}{\sum_{j \in\{\text { pilot_index }\}} j^{2}}
$$

where $\hat{\alpha}_{i}$ is the estimated slope. Also $i$ and $j$ represent indices for OFDM symbol and subcarrier, respectively. The slope estimation optimized in least mean squared sense utilizing channel quality, payload data and the pilot can be easily derived as follows:

$$
\hat{\alpha}_{i}=\frac{\sum_{j \in\{\text { plot_index }\}}\left|\hat{H}_{j}\right|^{2} \cdot j \cdot \phi_{i, j}+\sum_{j \in\{\text { data_index }\}}\left|\hat{H}_{j}\right|^{2} \cdot j \cdot \phi_{i, j}^{\prime}}{\sum_{j \in\{\text { pilot_index }\}}\left|\hat{H}_{j}\right|^{2} \cdot j^{2}+\sum_{j \in\{\text { data_index }\}}\left|\hat{H}_{j}\right|^{2} \cdot j^{2}}
$$

Here the squared channel gain for the corresponding subcarriers is used for the estimation which is expected to improve the estimation and tracking performances.

\section{Simulation Results}

In this section four representative tracking schemes are compared based on the BER performances. In all cases the maximum offset 40ppm is assumed which the IEEE 802.11a/g system allows. Also the packet structure follows the above mentioned high speed wireless LAN system with 500 byte payload data per packet modulated with BPSK. The RMS delay spread value is assumed to be 50ns. The pilot based scheme shows $1.9 \mathrm{~dB}$ degradation and 'Decision + Pilot' case $0.7 \mathrm{~dB}$ compared to the ideal performance. On the other hand the performance of 'Decision + Pilot + CSI' shows comparable performance to the ideal one. As the simulation results show, the channel state information (CSI) is very effective in the fading channels (additional 1.0dB over the 'Decision + Pilot'). The performance advantage of the payload data based scheme over conventional pilot based one is about $1.0 \mathrm{~dB}$ gain as shown in Fig. 1. 


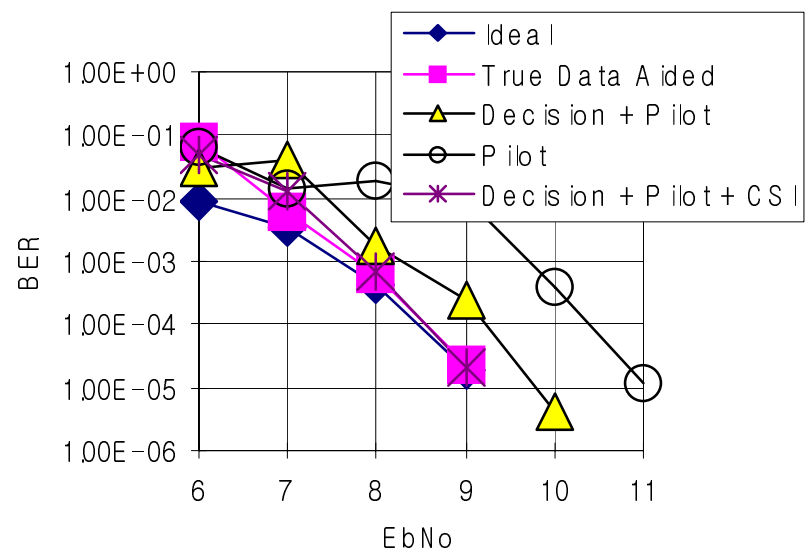

Fig. 1. BER simulation results for 50ns delay spread channel

\section{Conclusion}

This paper proposes an improved phase tracking algorithm where both carrier frequency offset and the sampling frequency offset are estimated and tracked to reduce the packet error rate which is one of the major QoS factors for high capacity multimedia wireless network. In the proposed method the estimation reliability is improved by adopting channel gain weight or channel information on the phase error estimation process. Computer based simulation results are also discussed to verify the effectiveness of the scheme.

\section{Acknowledgements}

This work was supported by Material and Component R\&D program funded by Ministry of Commerce, Industry and Energy of Korea (under grant 10027927).

\section{References}

1. J. A. C. Bingham: Multicarrier modulation for data transmission: An idea whose time has come. IEEE Communication Magazine, vol. 28 (1990) 4-14

2. B. Yang, K. Letaief, R. Cheng, Z. Cao: Timing Recovery for OFDM Transmission. IEEE JSAC, vol. 8, no. 11 (2000) 2278-2291

3. J. Terry, J. Heiskala: OFDM Wireless LAN, A Theoretical and Practical Guide. Sam Publishing (2002) 